\title{
Forced primer selection modulates HIV-I replication and stability of the PBS
}

\author{
$\mathrm{Na} \mathrm{Ni*}$ and Casey D Morrow
}

Address: Department of Cell Biology, University of Alabama at Birmingham, Birmingham, Alabama, 35294, USA

* Corresponding author

from 2006 International Meeting of The Institute of Human Virology

Baltimore, USA. 17-2I November, 2006

Published: 21 December 2006

Retrovirology 2006, 3(Suppl I):P45 doi:10.1 I86/I742-4690-3-SI-P45

(C) $2006 \mathrm{Ni}$ and Morrow; licensee BioMed Central Ltd.

HIV-1 exclusively selects tRNALys3 as the primer to initiate the reverse transcription. To further study the mechanism of HIV-1 primer selection, the primer binding site (PBS) was modified to pair with tRNAPhe, tRNATyr, tRNAThr or tRNASer. The PBS stability and replication of these mutants were studied in the SupT1 and peripheral blood mononuclear cells (PBMC). Virus with PBS complementary to tRNAThr grew slightly slower than the wild type virus and maintained the PBS for extended culture time but ultimately reverted to use tRNALys 3 . In contrast, viruses with the PBS complementary to tRNAPhe or tRNASer rapidly reverted to utilize tRNALys3 during growth in SupT1 and PBMCs. HIV-1 with the PBS complementary to tRNATyr had severely compromised infectivity and did not grow in SupT1 or PBMCs. Previous studies have shown additional changes 5 ' to the PBS in U5 (the Aloop region) so as to be complementary to the anticodon of certain tRNAs allow HIV-1 to stably use these tRNAs for replication. The A-loop mutation stabilized the continued use of tRNAThr as primer during replication in SupT1 and PBMCs. In contrast, the utilization of tRNASer was not stabilized by A-loop mutation. The A-loop mutation severely impaired the replication of virus with PBS complementary to tRNAPhe and could not rescue the virus with PBS complementary to tRNATyr. The results of these studies demonstrate the forced selection of certain alternative primers can have different effects on HIV-1 replication and PBS stability. 